\title{
SIMNET: Simulation-based exercises for Computer Net- work Curriculum through Gamification and Augmented Reality
}

\author{
Alvaro Luis Fraga ${ }^{1}$, María Guadalupe Gramajo ${ }^{1}$, Federico Trejo $^{2}$, Selena Garcia ${ }^{2}$, \\ Gustavo Juarez ${ }^{2,3}$ and Leonardo Franco ${ }^{3}$ \\ ${ }^{1}$ Universidad Tecnológica Nacional, Facultad Regional Santa Fe \\ alvarofragalieee.org, gramajoguadalupe@gmail.com \\ ${ }^{2}$ Universidad Tecnológica Nacional, Facultad Regional Tucumán \\ \{federicotrejo, selenagarcialobo, juarez.gustavo\} @ieee.org \\ ${ }^{3}$ Universidad de Málaga, Depto. de Lenguajes y Ciencias de la Computación \\ lfrancollcc.uma.es
}

\begin{abstract}
Gamification and Augmented Reality techniques, in recent years, have tackled many subjects and environments. Its implementation can, in particular, strengthen teaching and learning processes in schools and universities. Therefore, new forms of knowledge, based on interactions with objects, contributing game, experimentation and collaborative work. Through the technologies mentioned above, we intend to develop an application that serves as a didactic tool, giving support in the area of Computer Networks. This application aims to stand out in simulated controlled environments to create computer networks, taking into account the necessary physical devices and the different physical and logical topologies. The main goal is to enrich the students' learning experiences and contribute to teacher-student interaction, through collaborative learning provided by the tool, minimizing the need for expensive equipment in learning environments.
\end{abstract}

Keywords: Gamification; Augmented Reality; Simulation-based exercises

\section{Introduction}

Economic inequality is a reality, it affects every sector of our society, and is mainly present in Third World countries. The immersion of graduates from public universities in third world countries becomes difficult, as they do not have the latest technologies or the necessary equipment to acquire the needed skills to perform effectively in real work problems. In the education sector, there are public institutions that do not have state-of-the-art technological elements or equipment that allow simulations or practices to be carried out in controlled environments for computer networks related careers, in contrast to private institutions or First World Countries. This scenario contributes negatively to students and graduates' employability.

Gamification and Augmented Reality (AR) techniques have positioned as attractive and interesting strategies to improve and innovate on many domains being education one of them. Applying them has strengthened the teaching and learning processes in schools and universities. Hence, it is possible to construct new forms of learning based 
on interactions with objects, providing games, experiments, challenges and collaborative work.

Gamification is defined as the use of game design elements and techniques, in nongame related contexts, to engage people and solve problems [1]. Implementing such techniques facilitates changes in the classic classroom activities, providing incentives for students through reward and scoring systems [2]. Using game mechanics in the classroom allows students to make mistakes and restart play, giving them the freedom to fail and experiment without fear [3].

In this sense, several researches that suggest capturing characteristics, capabilities, rules, and strategies provided by video game environments to promote creative thinking and lead to new ways of addressing real-world problems. Morschheuser et. al [4] describes as the concept of collaboration provided by the game dynamics through gamification techniques, influences the dynamics of the group, generating commitment, shared emotions, sense of belonging and definition of group norms. Game-based learning has been shown to have potential to modern students who are growing up in an era of interactive media and video games, making gambling in the classroom attractive and motivating [5]. Most studies highlight positive aspects of gamification, because they generate greater engagement and enjoyment, but these results often depend on the context of the gamified system and the characteristics of the player.

AR application shows potential in the education fields [6]. It can help to improve critical thinking skills, problem solving, and teamwork through different activities [7]. Students easily engage in the learning process and can enjoy the experience through game mechanics embedded in the AR activity. Because of AR many complex scenarios can be replicated and approached from a different perspective, such as physical and biological phenomena as well as astronomical phenomena.

Some of the advantages of this technology are the following: i) the equipment that students need is a cell phone and a pattern card; ii) the application can be used anywhere and anytime; iii) and can be developing to be used as a standalone application [8]. Therefore, AR has become a popular technology used in educational settings. In this role, AR has also turned into an important focus of research in recent years. One of the most important reasons AR technology is widely accepted because it no longer requires expensive hardware and sophisticated equipment. AR can be defined as a technology that superimposes virtual objects (increased components) in the real world. These virtual objects seem to coexist in the same space as real-world objects [9]. Several studies show that AR can improve the teaching and learning experience [10], [11].

The main objective of this research is to enrich students' learning experiences and improve the teacher-student interaction through collaborative learning. Therefore, a tool that allows the virtualization of controlled environments to create computer network infrastructure simulations challenges by combining gamification and AR techniques is presented in this contribution to achieve this objective.

This research also intends to be a support application to institution that do not have the budget to equip their laboratories with certain and necessary equipment to carry out the computer network practices.

The rest of the paper is structured as follows. Section 2 describes some related works with the application of gamification and AR techniques in the academic field. Section 
3 is divided in two subsections. The first one describes the guidelines needed to use SIMNET in classroom. The second part includes its architecture and description of the components used in the development of the application. Finally, section 4 shows the conclusions and future work.

\section{$2 \quad$ Related Works}

This section will mention some related works in the field. These studies have made use of the techniques we have mentioned in the introduction to this paper.

Ibáñez et. al [12] present a learning application that make use of AR technology to aboard the basic concepts of electromagnetism. This study also compared an AR-based application with an equivalent web-based application to study its learning effectiveness and the level of enjoyment of high school students. The analysis showed that the AR application led participants to achieve higher levels concentration than those achieved by web-based application users.

Quintero et. al [13] presented an AR application to promote spatial visualization in calculus courses for engineering students. The aim of this research was presenting to students a cognitive experience of the surface as the intersection of planes. This experience was driven for its first pilot test. The results concluded that the traditional approach of learning mathematics cannot remain unchanged and it is necessary new techniques that contribute to the students learning experience.

PhysicsPlayground [14] is an AR application that allows simulate physical phenomena. This application can be used by special equipment and was developed using PhysX API from NVIDIA. PhysicsPlayground helps to teach and learn mechanics. This tool allows students to construct virtual models to study physical properties, verify formulas, develop theories and actively take part in physical education, encouraging experimentation and understanding. Authors highlight the nearly haptic interaction when building and running experiments in the application. Also, authors remark the possibility to change parameters in real time to take new perspectives of the results.

AR facilitates the observation of events that cannot to be easily observed with the naked eye [11]. Therefore, this can be exploited to increase students' motivation and help them to acquire better research skills [15].

According to [16], AR most significant advantage is its "unique ability to create hybrid learning environments that combine digital and physical objects, facilitating the development of processing skills such as critical thinking, problem-solving and communication through interdependent collaborative exercises".

In summary, it is clear how AR and gamification have been useful for improving learning experiences, both to provide student another perspective from which to approach the comprehension of certain complex issues such as visualization of phenomena invisible to our eyes. It can also be useful to provide virtual scenarios in which the equipment is not available to carry them out in reality or to simulate a practice. That is why our proposal makes use of these technologies to develop an application that can provide the student with a new experience in computer networks. 
In the following section, we will describe the methodology to apply the application in the classroom and the components used to develop it.

\section{SIMNET: Methodology and Implementation}

This section will present the scenario adopted to make a pilot test of the application developed. It will describe the characteristics it provides, and the design chosen for its development.

\subsection{Usage Methodology}

Each one of these course assignments has a scenario description, an aim related to the specific exercise and a QR code. This code attached to each exercise allows interaction between the application and the assignment. Once every student will have been supplied with the application on their mobile devices, they will access the environment developed for the activity.

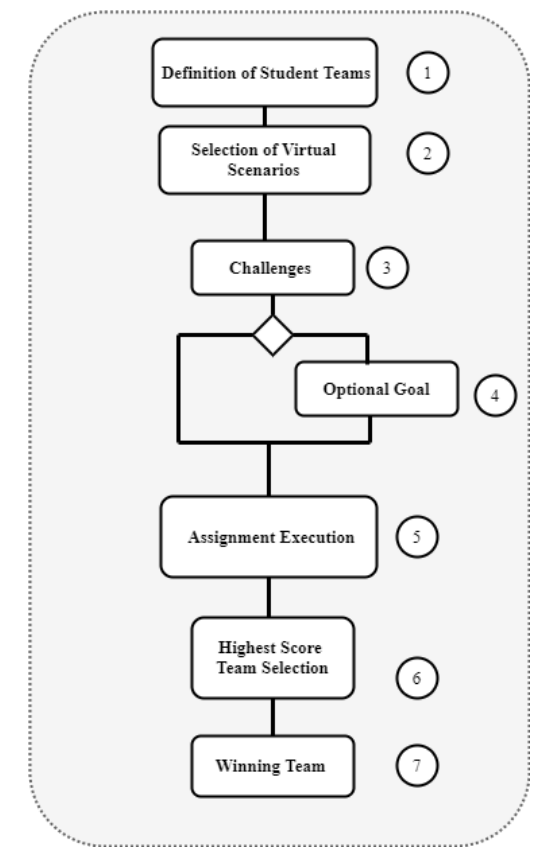

Fig. 1. Methodology workflow

The Figure 1 shows the activities flow to consider when using SIMNET. This workflow consists of 7 activities: Definition of student teams (1), Selection of virtual scenarios (2), Challenges (3), Optional goals (optional activity) (4), Assignment execution (5), Highest score team selection (6), Winning team (7). 
Initially, teachers will separate students into groups of up to 4 members to start the assignment as indicated in step (1). As soon as students have formed the teams and entered the application. The students will register the group and full name as it is shown in Figure 2a, to record the group to which each member belongs. This information is added to a spreadsheet in a cloud service. Teachers are the only ones that will be able to access to this spreadsheet. Then, they will scan the QR codes to begin the activity.

The activities will be related to complete a certain virtual implementation of a computer network as indicated in step (2). Using components pre-assigned for each exercise. The components will appear in the application's toolbox when the activity begins. Students will be able to aboard the problems which represents the challenges indicated in step (3), with different virtual components. Having multiple ways to solve each assignment, so optional objectives have been added. Optional objectives range from the use of a specific component to accomplish the task to the lowest possible use of economic resources, step (4). The teams that decide to solve them will get extra points in the final score.

An example of an activity is shown in Figure 2b, where students are encouraging to build a rack for certain number of personal computer. Next students must proceed with the assignment execution defined by the teacher using the application, step (5). The students have many switch options and type of connectors. Each activity has their component, information and cost expressed in dollars. After the student decides, which components will be use, they must select them and the components will be added to the rack and the cost of his solution will be display at the left corner of the screen.

Once the student achieves a possible solution to the problem. The application notifies them that a possible solution was found.
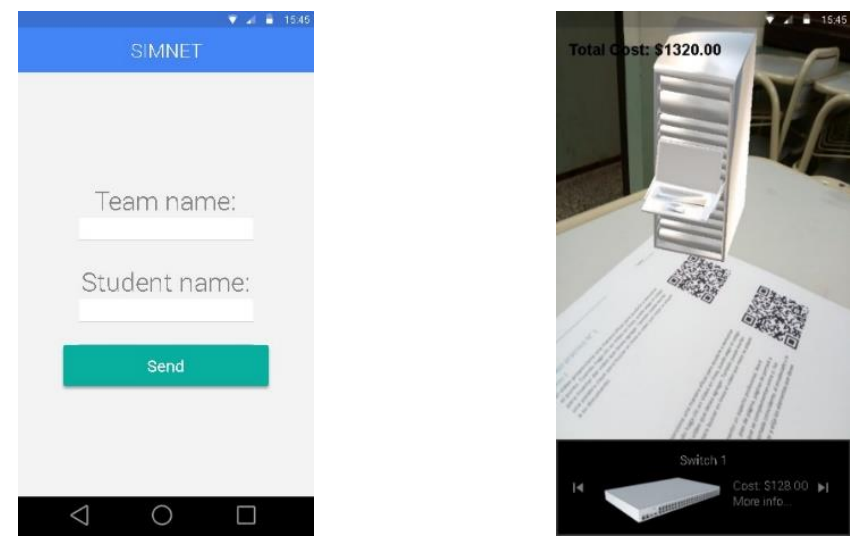

Fig. 2. a) Screenshot of the application main screen; b) Screenshot of the application in use.

These activities encourage teamwork and cooperative work bringing together the above-mentioned benefits of using gamification. Every member of a team also can submit their own answer, but at the end of the assignment, only the best score will be taken as the final mark. 
Next, the teacher arranges the results using the spreadsheet from the cloud service, a portion of the spreadsheet is exemplified in Table 1, as indicated in step (6) and finally lists the winning team, step (7).

Table 1. Portion of Student Registration Sheet

\begin{tabular}{|c|c|c|c|c|}
\hline Group Name & Student Name & Exercise 1 & Exercise 2 & Exercise 3 \\
\hline Team 1 & Student 1 & $\$ 3250$ & $\$ 2050$ & $\$ 1099$ \\
\hline Team 1 & Student 2 & $\$ 3250$ & $\$ 2050$ & $\$ 1099$ \\
\hline Team 2 & Student 4 & $\$ 4000$ & $\$ 2200$ & $\$ 1300$ \\
\hline
\end{tabular}

\subsection{Implementation}

This subsection describes the components used to develop the prototype of AR application.

We have designed and developed the first version of this proposal targeting mobile devices with an Android operating system up to version 4.4. This application makes use of the Qualcomm Vuforia SDK framework, jMonkeyEngine() and the Android platform.

Qualcomm Vuforia SDK software requires a version 3 or higher of the operative system to ensure that the application will work. The Vuforia SDK includes cutting-edge computer vision algorithms aimed at recognizing and tracking an increased variety of objects, including markers, target images, 3D objects, among others for any user developing the software. Although they are unnecessary for the development of sensor-based AR applications, they allow for easy deployment of computer vision-based AR applications.

$\mathrm{j}$ MonkeyEngine is a free gaming engine that brings the $3 \mathrm{D}$ graphics of programs to life. It provides intermediate software for 3D graphics and games that frees you from the exclusive coding in low-level OpenGL, for example, by providing an asset system for importing models, pre-defined lighting, physics and special effects modules that are not embedded in 3D objects.

The Android development toolkit includes the Eclipse Integrated Development Environment (IDE), the Android development toolkit for Eclipse (ADT) and many versions of the Android operative system. Specifically, to effectively use these technologies, it is necessary for the device to have a rear camera, GPS module, and gyroscope, and accelerometers and compass.

An AR application can be structured in three layers:

- Upper layer: Contains the domain logic, also known as the application layer.

- Intermediate layer: Modules or components that enable the use of AR, such as visualization, tracking, recording, and interaction.

- Lower layer: Conformed by the operative system, this layer integrates the components providing interfaces to manage all resource of the device. 


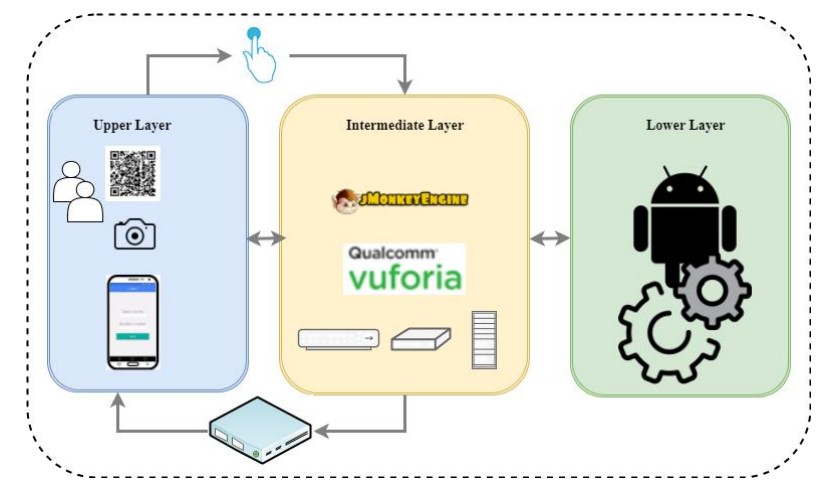

Fig. 4. SIMNET Architecture Schema.

After determining the architecture and its components, it is necessary to define how information flows in a typical AR application. In recent years, developers have converged on the well-used method of combining these tools using a similar order of execution, the AR control flow or rather the AR control loop. This diagram, Figure 4, should be read from the left to right. Displays the main sequence of tasks of an AR application, which is repeated in a cycle.

First, the camera is accessed, and the current frame is retrieved and displayed. Then, depending on the AR, sensor data is retrieved, or the camera frame is processed with Computer Vision algorithms to search and track objects. Afterward, virtual objects are transformed and moved into the virtual world to match the real world. Finally, user interactions and gestures are processed to further change virtual objects or perform domain-specific tasks. At the same time, it is necessary to add domain-specific logic and other activities related to the operating system.

\section{Conclusion}

Augmented reality allows the students interaction between the real and virtual worlds. The capabilities AR of merging virtual and real worlds together have given birth to new approaches that allow for improving the quality of learning. The effectiveness of AR can be further extended when it combines with other types of techniques such as gamification. Gamification improves the student's engagement, motivation, and performance when carrying out a certain task; it does so by incorporating game mechanics and elements, thus making that task more attractive. Combining both techniques can generate great benefits in the educational area, allowing to experience new ways of learning, generate interest in the students and provide new skills that facilitate their insertion into the workplace.

For this reason, we presented this tool to simulate computer networks challenges in a collaborative environment that allows interaction with virtual devices, through an application that combines the advantages of AR and gamification techniques.

In future works, authors will present a pilot experience using the application in the computer network course starting next year. 
Acknowledgments. LF and GEJ acknowledge support from grant TIN2014-8516C2-1-R from MICINN-SPAIN which includes FEDER funds, and from the Universidad de Málaga (España) through “Ayudas Plan Propio".

\section{References}

[1] S. Deterding, D. Dixon, R. Khaled, and L. Nacke, "From game design elements to gamefulness: defining gamification," in Proceedings of the 15th international academic MindTrek conference: Envisioning future media environments, 2011, pp. 9-15.

[2] M. D. Hanus and J. Fox, "Assessing the effects of gamification in the classroom: A longitudinal study on intrinsic motivation, social comparison, satisfaction, effort, and academic performance," Comput. Educ., vol. 80, pp. 152-161, 2015.

[3] L. De-Marcos, A. Dom'linguez, J. Saenz-de-Navarrete, and C. Pagés, “An empirical study comparing gamification and social networking on e-learning," Comput. Educ., vol. 75, pp. 82-91, 2014

[4] B. Morschheuser, M. Riar, J. Hamari, and A. Maedche, "How games induce cooperation? A study on the relationship between game features and we-intentions in an augmented reality game," Comput. Human Behav., vol. 77, pp. 169-183, 2017.

[5] I. Glover, "Play as you learn: gamification as a technique for motivating learners," in Proceedings of the 2013 World Conference on Educational Multimedia, Hypermedia and Telecommunications (Edmedia 2013), 2013.

[6] N. F. Saidin, N. D. A. Halim, and N. Yahaya, "The Potential of Augmented Reality Technology in Education: A Review of Previous Research.". in Proceedings of the International Graduate Conference on Engineering, Science and Humanities (IGCESGH). UTM. 2014.

[7] D. N. E. Phon, M. B. Ali, and N. D. A. Halim, "Collaborative augmented reality in education: A review," in Teaching and Learning in Computing and Engineering (LaTiCE), 2014 International Conference on, 2014, pp. 78-83.

[8] L. B. Kiat, M. B. Ali, N. D. A. Halim, and H. B. Ibrahim, "Augmented Reality, Virtual Learning Environment and Mobile Learning in education: A comparison," in e-Learning, e-Management and eServices (IC3e), 2016 IEEE Conference on, 2016, pp. 23-28.

[9] R. Azuma, Y. Baillot, R. Behringer, S. Feiner, S. Julier, and B. MacIntyre, "Recent advances in augmented reality,” IEEE Comput. Graph. Appl., vol. 21, no. 6, pp. 34-47, 2001.

[10] P. S. Medicherla, G. Chang, and P. Morreale, "Visualization for increased understanding and learning using augmented reality," in Proceedings of the international conference on Multimedia information retrieval, 2010, pp. 441-444.

[11] H.-K. Wu, S. W.-Y. Lee, H.-Y. Chang, and J.-C. Liang, "Current status, opportunities and challenges of augmented reality in education," Comput. Educ., vol. 62, pp. 41-49, 2013.

[12] M. B. Ibáñez, Á. Di Serio, D. Villarán, and C. D. Kloos, "Experimenting with electromagnetism using augmented reality: Impact on flow student experience and educational effectiveness," Comput. Educ., vol. 71, pp. 1-13, 2014.

[13] E. Quintero, P. Salinas, E. González-Mendivil, and H. Ramirez, “Augmented reality app for calculus: A proposal for the development of spatial visualization,” Procedia Comput. Sci., vol. 75, pp. 301-305, 2015.

[14] H. Kaufmann and B. Meyer, Simulating educational physical experiments in augmented reality. ACM, 2008.

[15] S. Sotiriou and F. X. Bogner, "Visualizing the invisible: augmented reality as an innovative science education scheme," Adv. Sci. Lett., vol. 1, no. 1, pp. 114-122, 2008.

[16] M. Dunleavy, C. Dede, and R. Mitchell, "Affordances and limitations of immersive participatory augmented reality simulations for teaching and learning," J. Sci. Educ. Technol., vol. 18, no. 1, pp. 7$22,2009$. 